Supporting Information

\title{
A Simple Drop-and-Dry Approach to Grass-Like Multifunctional Nano-Coating on Flexible Cotton Fabrics using In Situ Generated Coating Solution Comprising Titanium-Oxo Clusters and Silver Nanoparticles
}

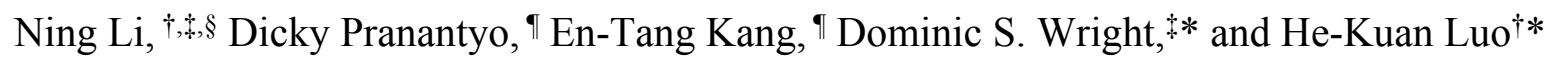

$\dagger$ Institute of Materials Research and Engineering, Agency for Science, Technology and Research, 2 Fusionopolis Way, \#08-03, Innovis, Singapore 138634

$\$$ Chemistry Department, University of Cambridge, Lensfield Road, Cambridge, CB2 1EW, UK

" Department of Chemical \& Biomolecular Engineering, National University of Singapore, Kent Ridge, Singapore 119260

$\S$ Present Address: NanoBio Lab, 31 Biopolis Way, \#09-01 The Nanos, Singapore 138669 

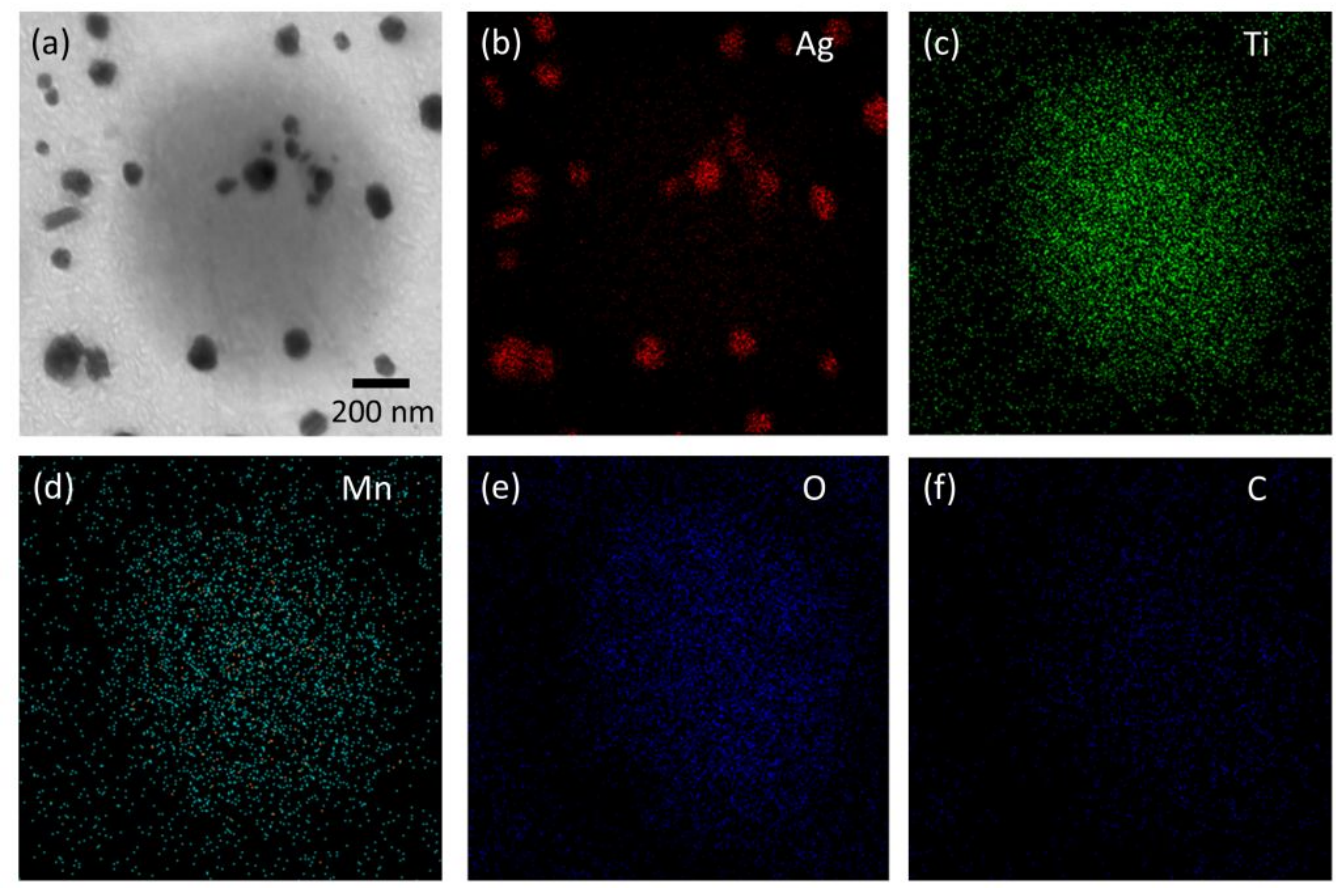

Figure S1. TEM image (a) and EDX mapping for (b) Ag, (c) Ti, (d) Mn, (e) O, and (f) C, done by drop-casting the precursor solution directly onto the $\mathrm{Cu}$ grid.

(a)

(b)
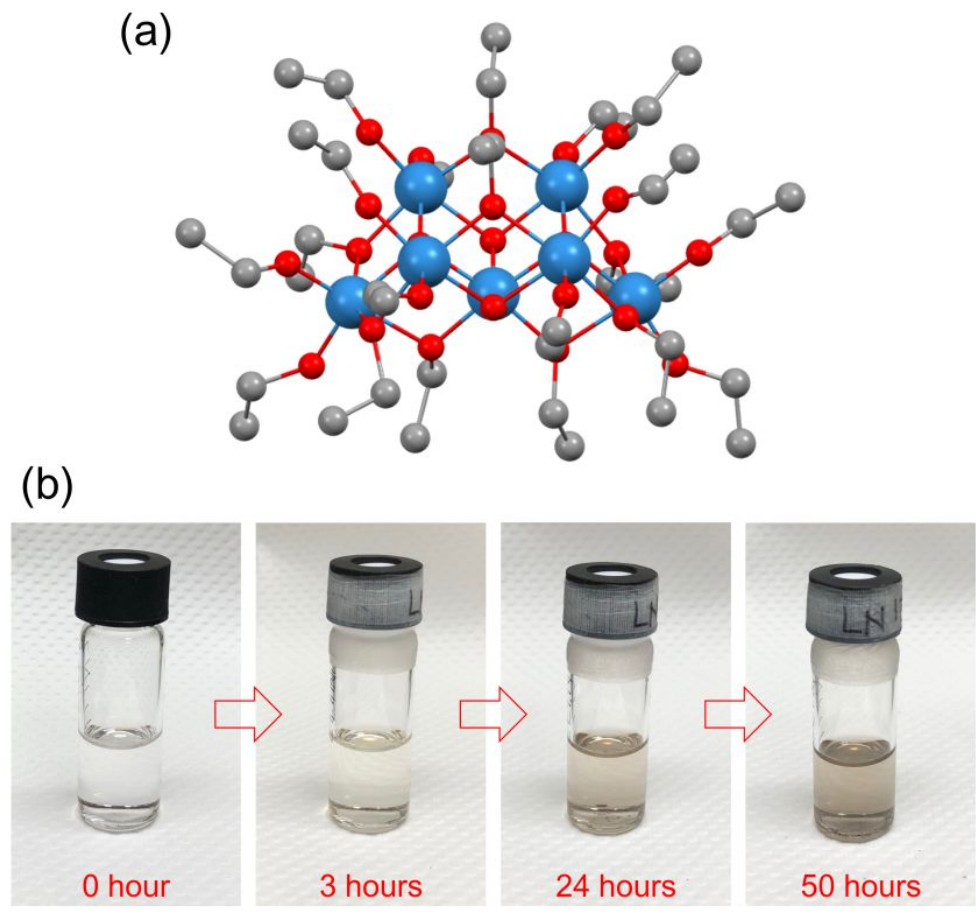

Figure S2. (a) Ball-stick representation of TOC $\left[\mathrm{Ti}_{7} \mathrm{O}_{4}(\mathrm{OEt})_{20}\right]$. Color code for atoms: $\mathrm{Ti}=$ cyan, $\mathrm{O}=$ red, $\mathrm{C}=$ gray. Hydrogen atoms are omitted for clarity. (b) Digital photographs of the solution containing $10 \mathrm{mg} \mathrm{mL}^{-1} \mathrm{AgNO}_{3}$ and $2.5 \mathrm{mg} \mathrm{mL}^{-1}\left[\mathrm{Ti}_{7} \mathrm{O}_{4}(\mathrm{OEt})_{20}\right]$ in the binary solvent of toluene and methanol ( $3: 2$ volume ratio) under ambient conditions. Color change from almost colorless to dark brown was seen, confirming the formation of colloidal AgNPs. 


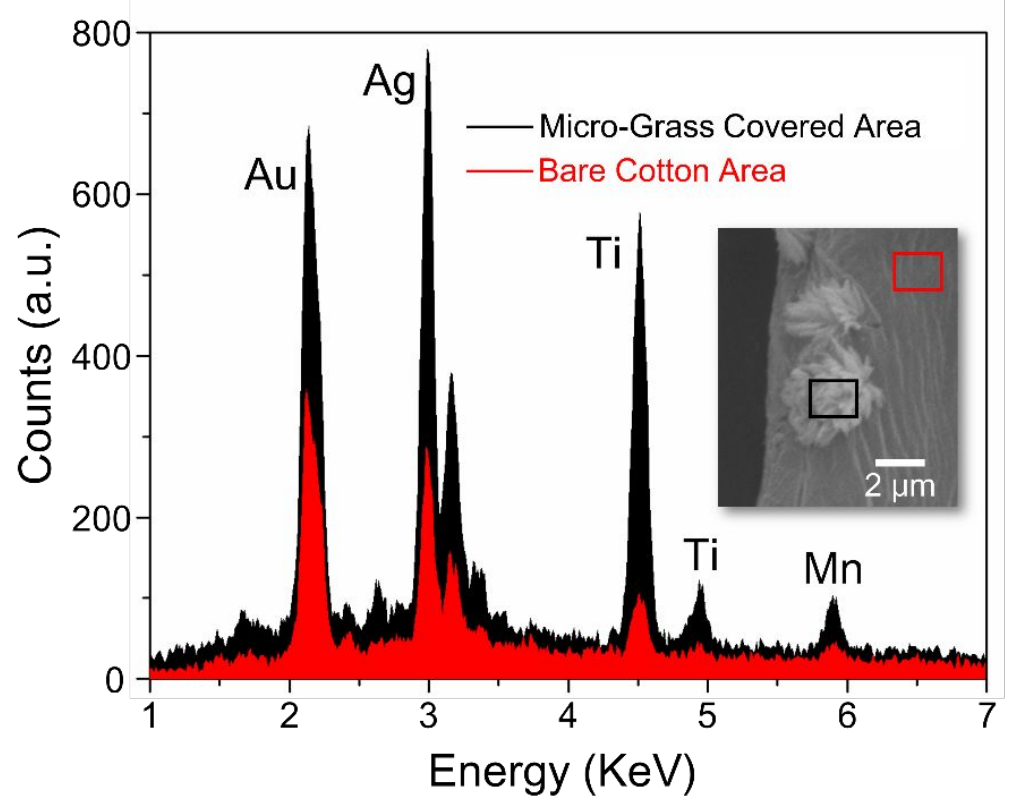

Figure S3. FESEM-EDX spectra of $\mathbf{1}|\mathbf{A g N P s}|$ Cotton
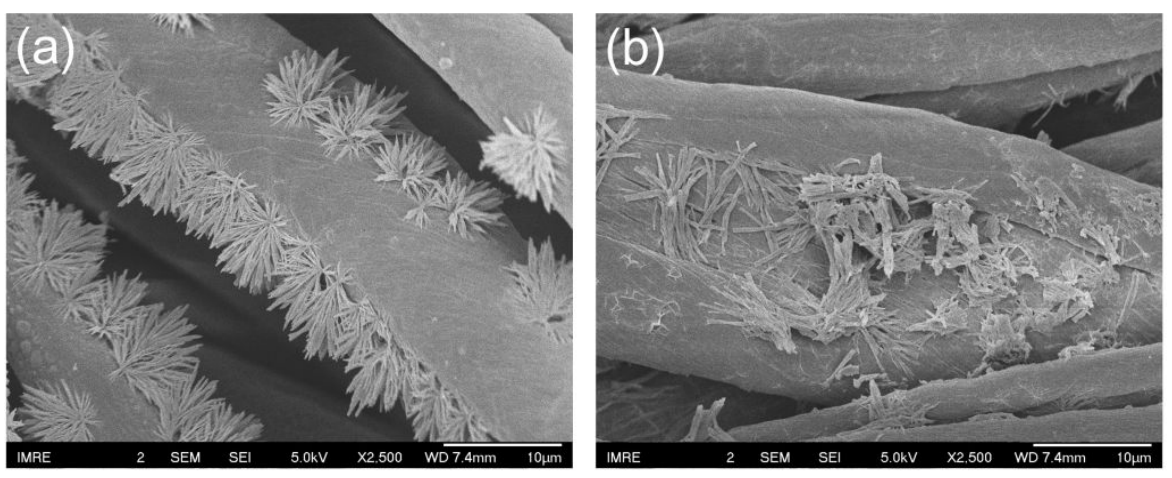

Figure S4. FESEM images of in situ generated surface morphology by drop-casting the precursor solution in (a) argon and (b) air.
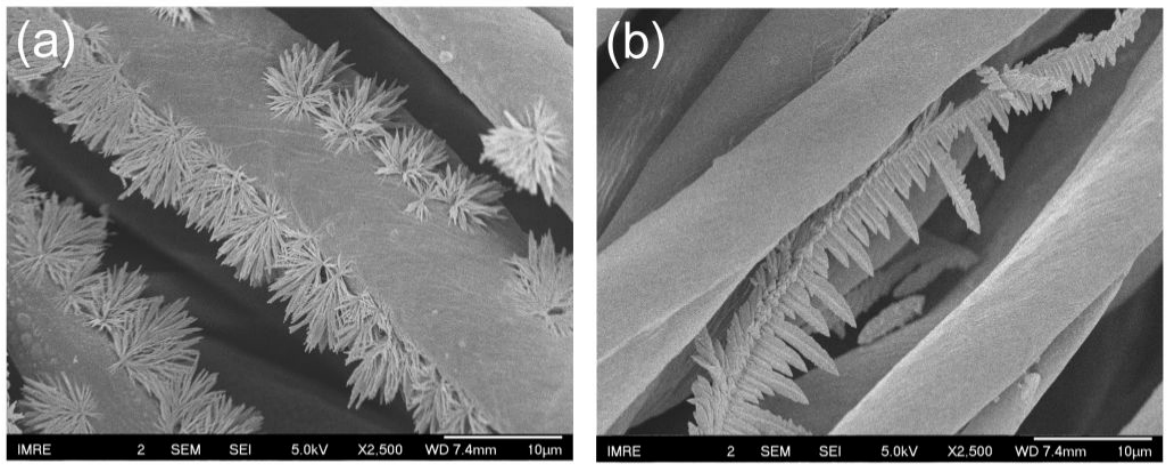

Figure S5. FESEM images of in situ generated surface morphology by drop-casting the precursor solution containing $\mathrm{AgNO}_{3}$ and (a) TOC $\left[\mathrm{Ti}_{18} \mathrm{MnO}_{30}(\mathrm{OEt})_{20}(\mathrm{MnPhen})_{3}\right]$, (b) TOC $\left[\mathrm{Ti}_{7} \mathrm{O}_{4}(\mathrm{OEt})_{20}\right]$ in argon. 

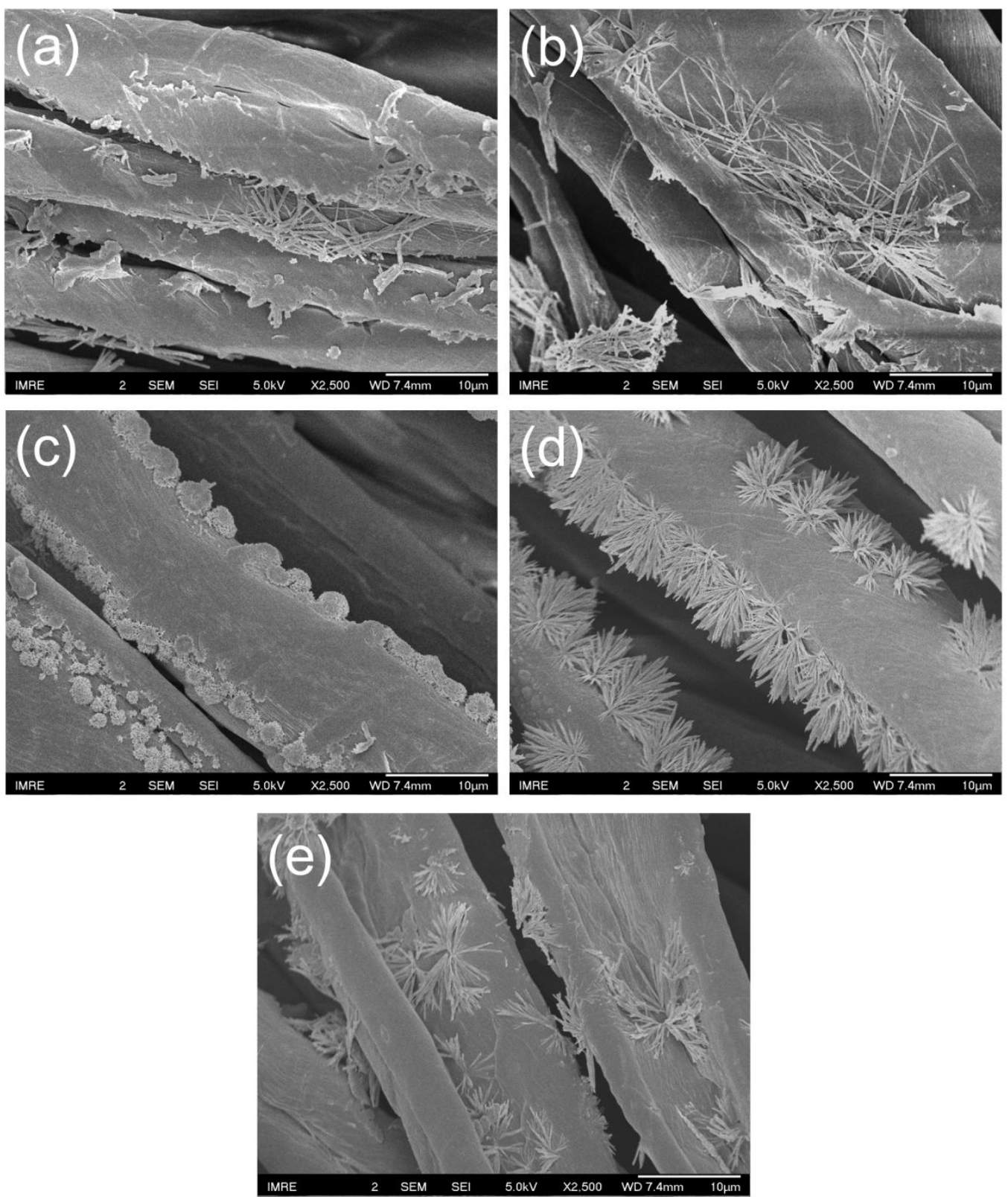

Figure S6. FESEM images of in situ generated surface morphology by drop-casting the precursor solution containing $\mathrm{AgNO}_{3}$ at concentrations of (a) $1.25 \mathrm{mg} \mathrm{mL}^{-1}$, (b) $2.5 \mathrm{mg} \mathrm{mL}^{-1}$, (c) $5.0 \mathrm{mg} \mathrm{mL}^{-1}$, (d) $10 \mathrm{mg} \mathrm{mL}^{-1}$ and (e) $15 \mathrm{mg} \mathrm{mL}^{-1}$, with the TOC 1 concentration fixed at $2.5 \mathrm{mg} \mathrm{mL}^{-1}$. 

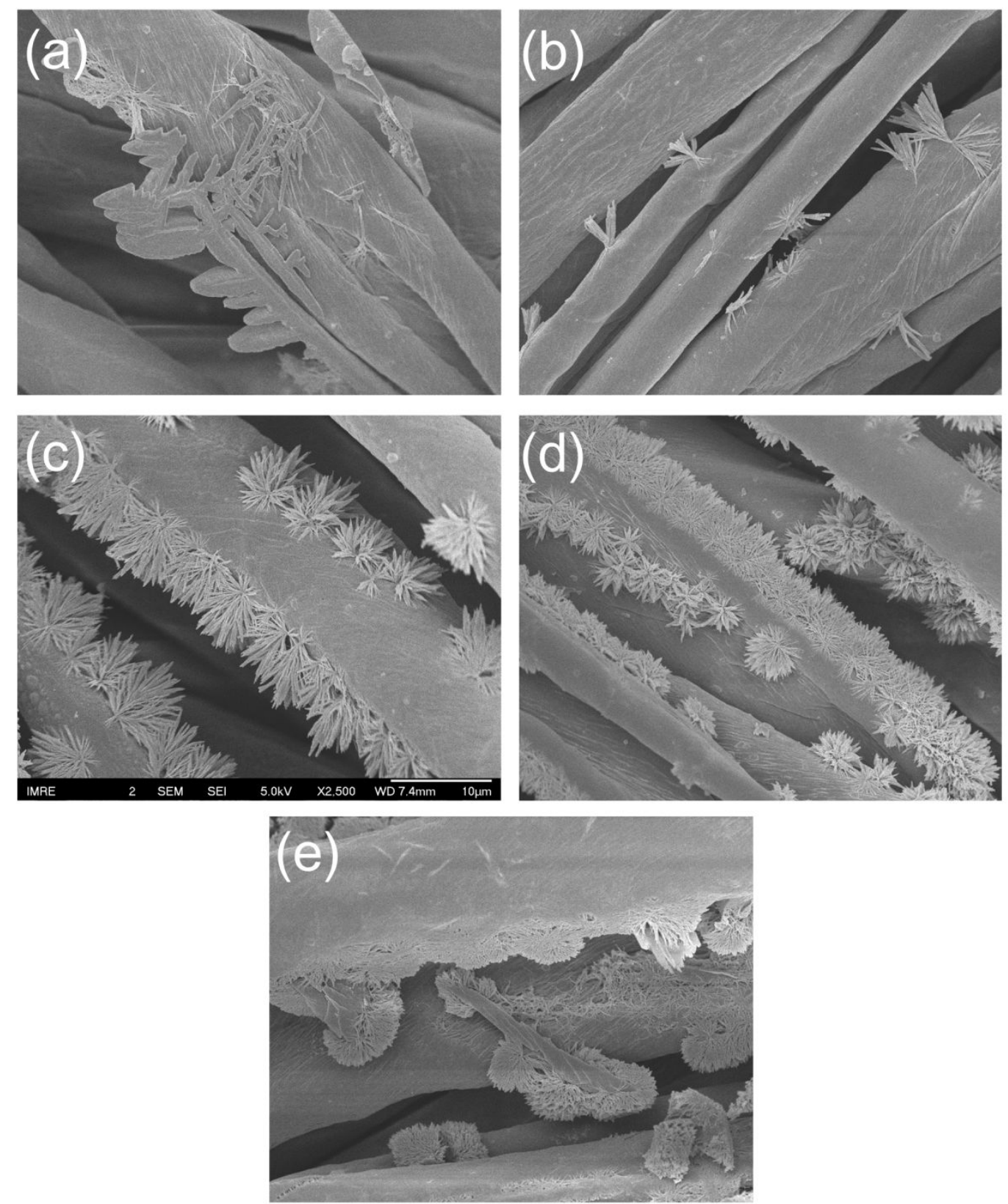

Figure S7. FESEM images of in situ generated surface morphology by drop-casting the precursor solution containing TOC 1 at concentrations of (a) $0.625 \mathrm{mg} \mathrm{mL}^{-1}$, (b) $1.25 \mathrm{mg} \mathrm{mL}^{-}$ 1, (c) $2.5 \mathrm{mg} \mathrm{mL}^{-1}$, (d) $5.0 \mathrm{mg} \mathrm{mL}^{-1}$ and (e) $7.5 \mathrm{mg} \mathrm{mL}^{-1}$, with the $\mathrm{AgNO}_{3}$ concentration fixed at $10 \mathrm{mg} \mathrm{mL}^{-1}$. 

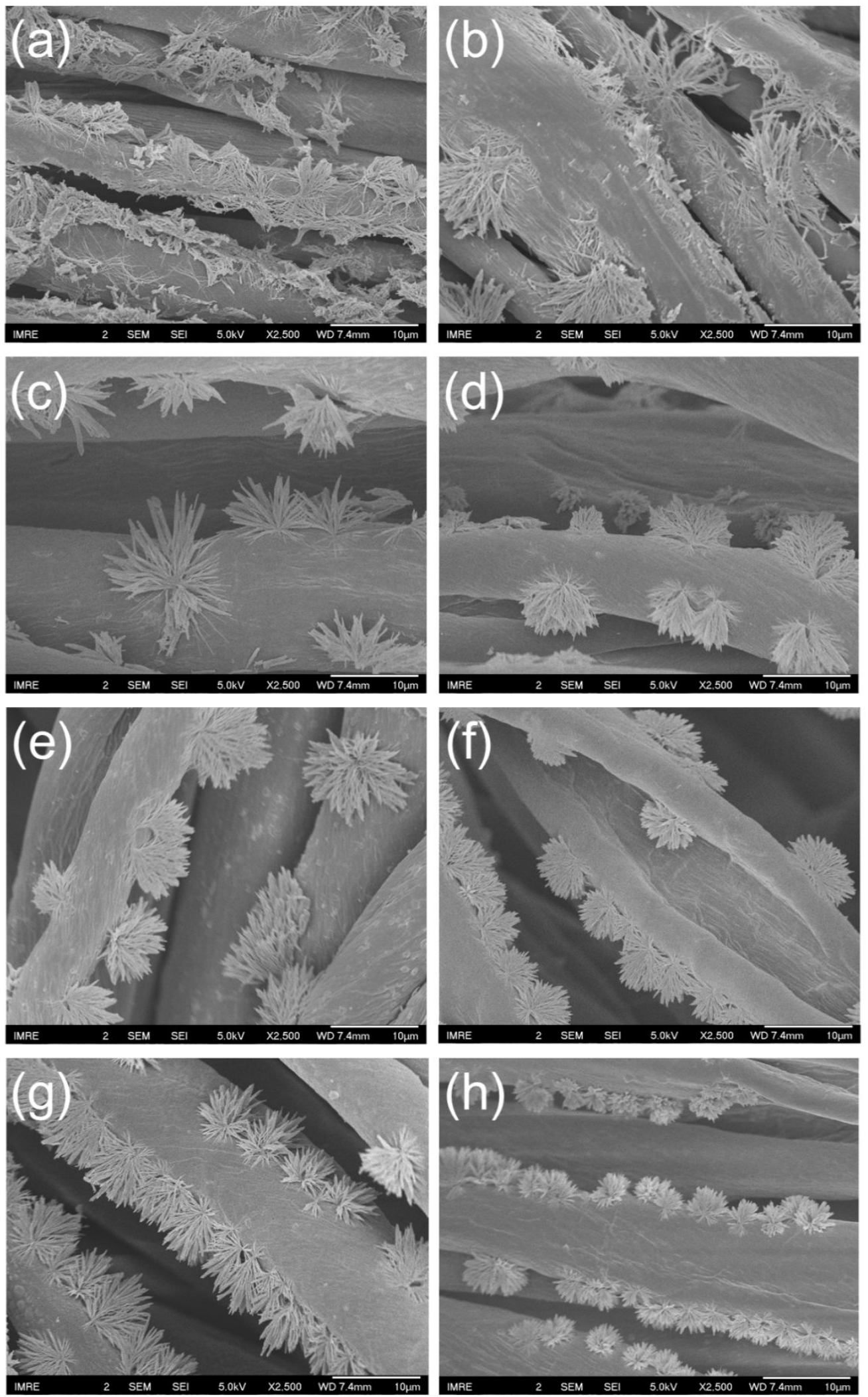

Figure S8. FESEM images of in situ generated surface morphology by drop-casting the precursor solution containing $10 \mathrm{mg} \mathrm{mL}^{-1} \mathrm{AgNO}_{3}$ and $2.5 \mathrm{mg} \mathrm{mL}^{-1}$ TOC 1 after an incubation period of (a) $0 \mathrm{~h}$, (b) $1 \mathrm{~h}$, (c) $3 \mathrm{~h}$, (d) $5 \mathrm{~h}$, (e) $9 \mathrm{~h}$, (f) $20 \mathrm{~h}$, (g) $24 \mathrm{~h}$ and (h) $48 \mathrm{~h}$. 

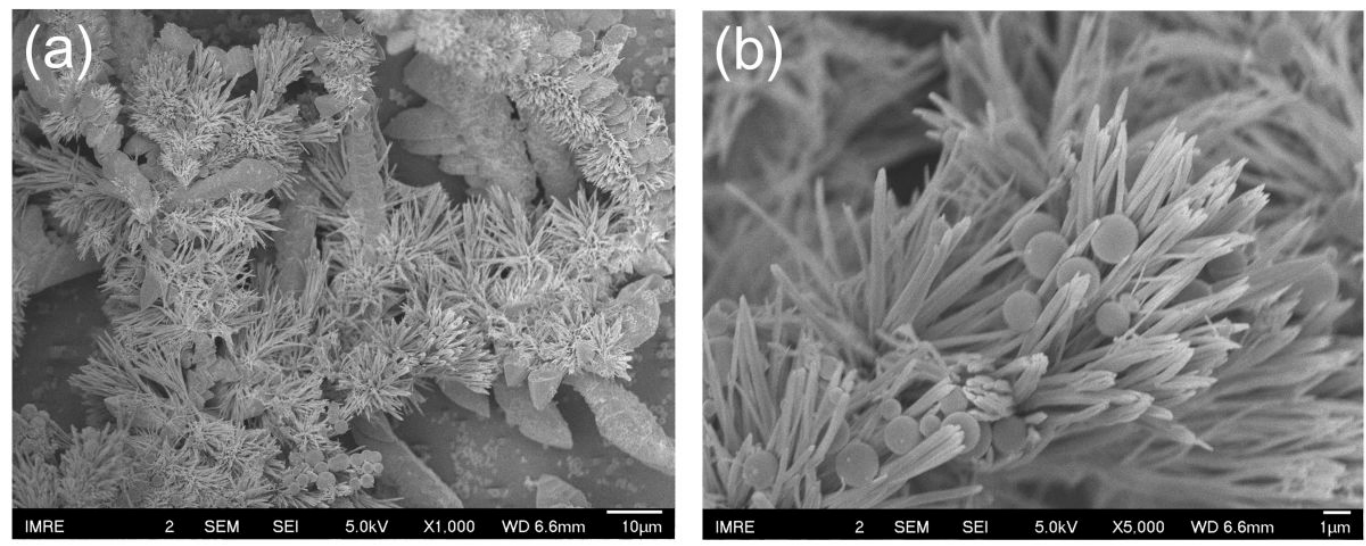

Figure S9. FESEM images of in situ generated surface morphology by drop-casting the precursor solution on glass substrate in argon atmosphere: (a) low and (b) high magnification.
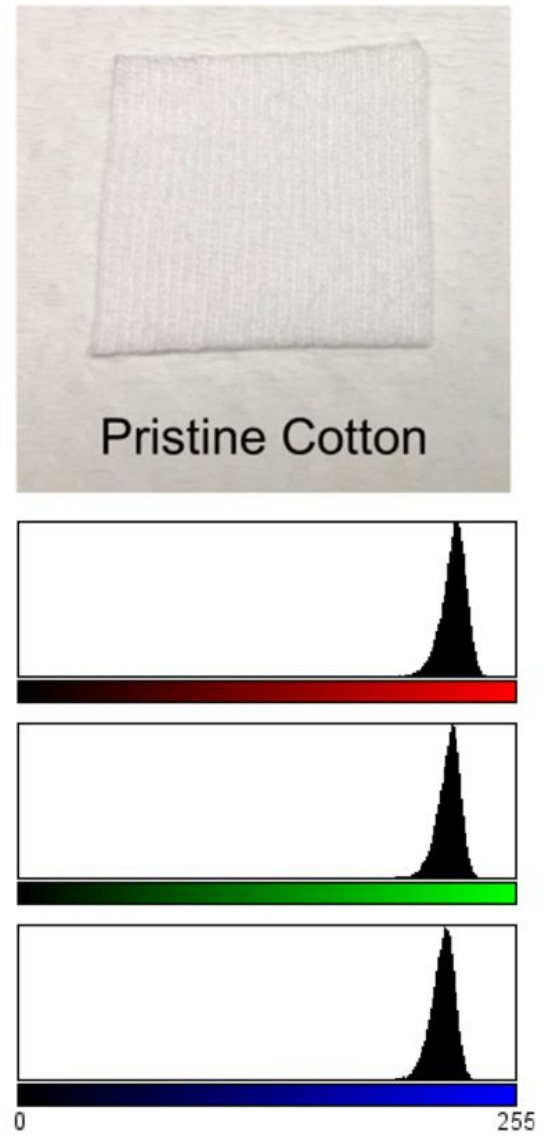

Count 100464

rMean: 223.62 ISD: $6.94 \quad$ rMode: 225 gMean: 221.02 gSD: 6.55 gMode: 223 bMean: 217.91 bSD: $6.67 \quad$ bMode: 219
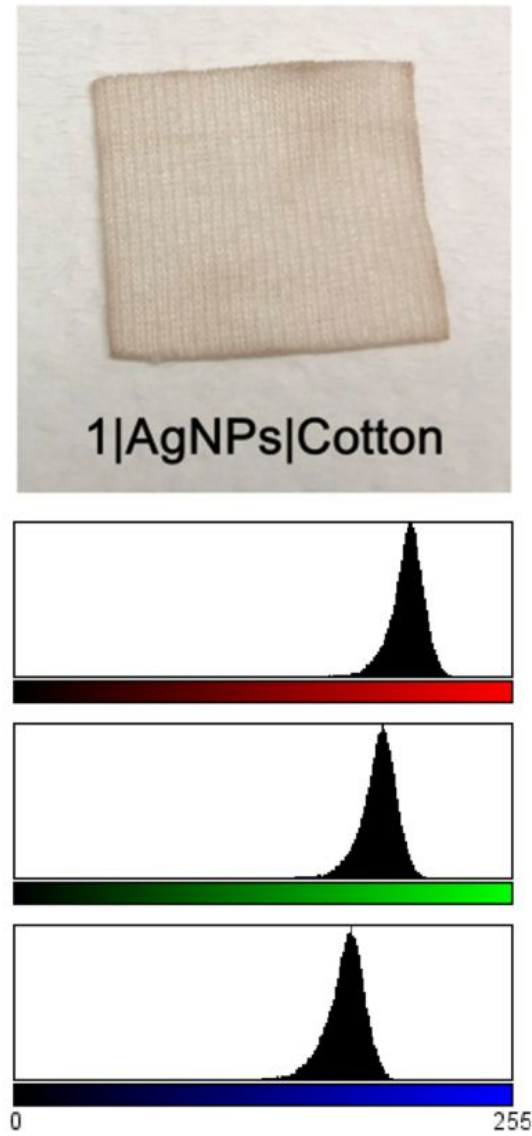

Count: 100763

rMean: $201.50 \quad$ rSD: $9.34 \quad$ rMode: 204

gMean: 186.80 gSD: 10.09 gMode: 189

bMean: 169.71 bSD: 10.76 bMode: 173

Figure S10. Digital photographs of pristine cotton (left) and $1|\operatorname{AgNPs}|$ Cotton (right). The physical dimension is $2 \mathrm{~cm}$ by $2 \mathrm{~cm}$ for each cotton fabric swatch. Their color histogram and RGB values generated from ImageJ are also shown at the bottom. 


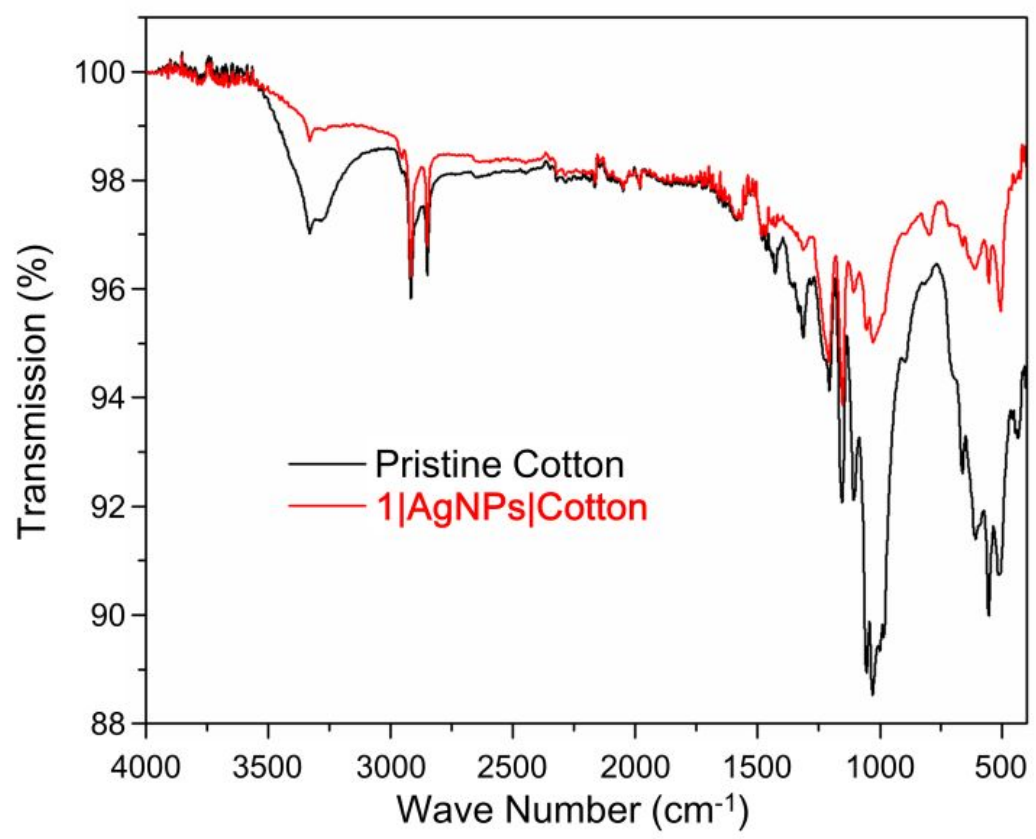

Figure S11. FTIR-ATR spectra of pristine cotton and $\mathbf{1}|\mathbf{A g N P s}| \mathbf{C o t t o n}$

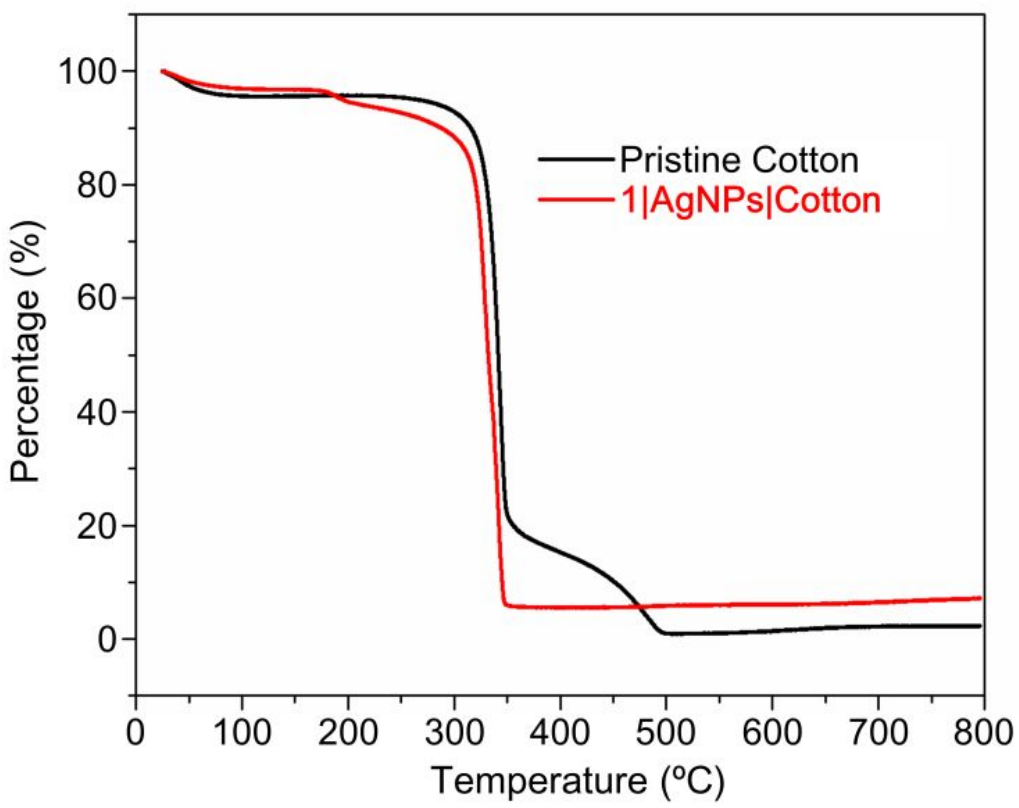

Figure S12. TGA profile of pristine cotton and $\mathbf{1}|\mathbf{A g N P s}|$ Cotton in air flow of $60 \mathrm{~mL} \mathrm{~min}-1$ 


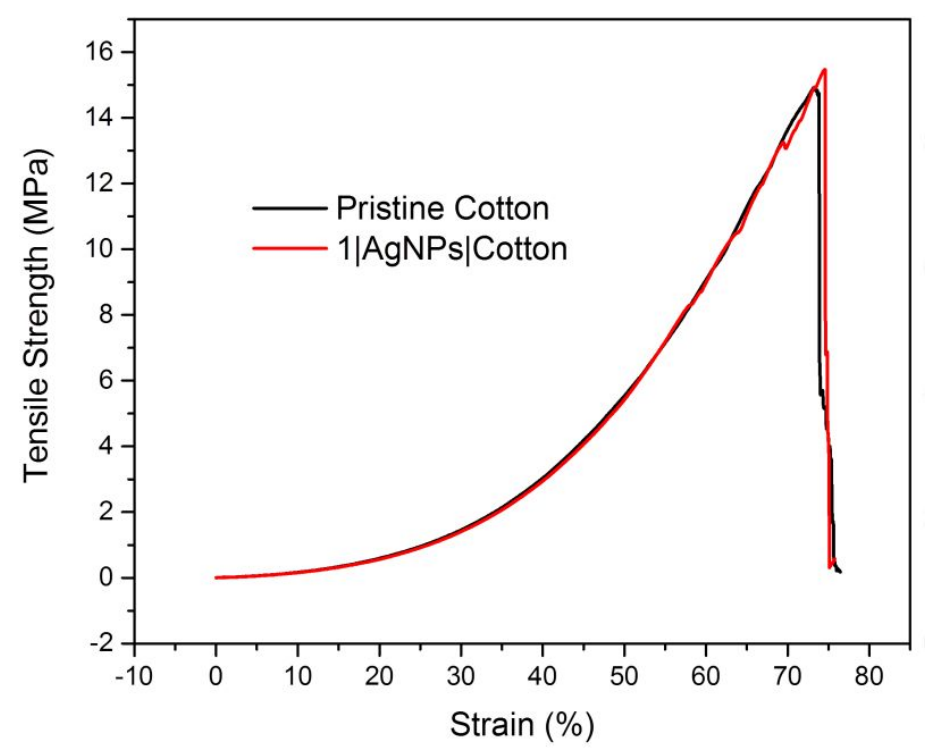

Figure S13. Tensile strength measurement of $\mathbf{1}|\mathbf{A g N P s}|$ Cotton and pristine cotton fabrics

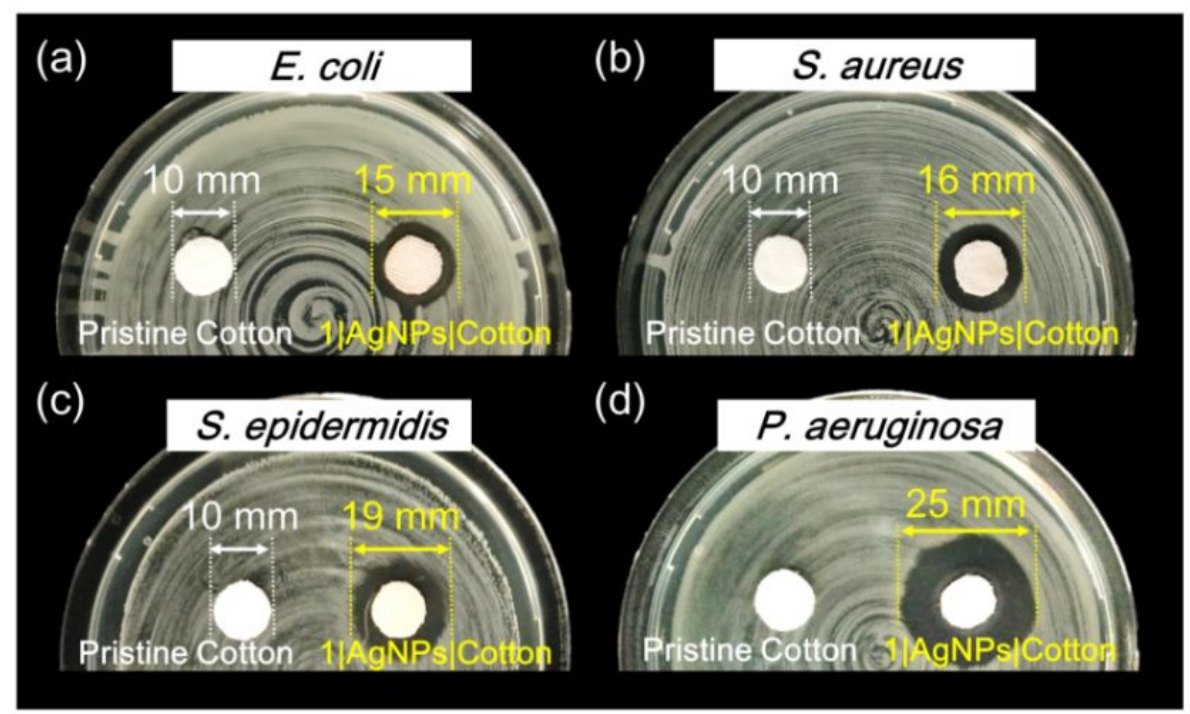

Figure S14. Digital photographs showing the antibacterial activity of $1|\operatorname{AgNPs}|$ Cotton in dark against (a) E. coli, (b) S. aureus, (c) S. epidermidis, and (d) P. aeruginosa. The samples used are all $10 \mathrm{~mm}$ in diameter and the size of the inhibition zones are indicated for comparison. 

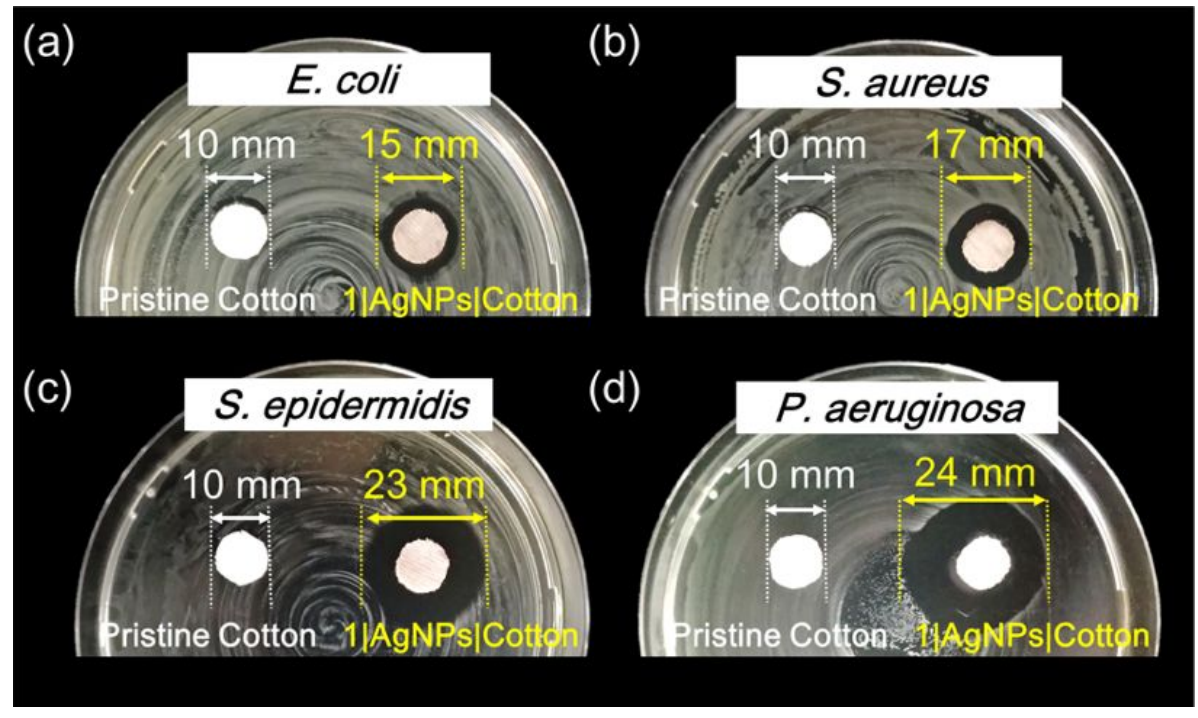

Figure S15. Digital photographs showing the antibacterial activity of the washed 1|AgNPs|Cotton sample against (a) E. coli, (b) $S$. aureus, (c) S. epidermidis, and (d) $P$. aeruginosa in dark conditions. The samples used are all $10 \mathrm{~mm}$ in diameter and the size of the inhibition zones are indicated for comparison.

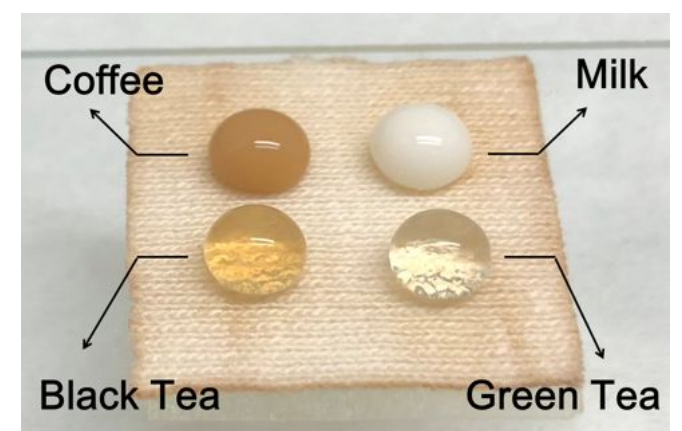

Figure S16. Digital photograph showing the hydrophobicity of $\mathbf{1}|\mathbf{A g N P s}| \mathbf{C o t t o n}$ against common beverages

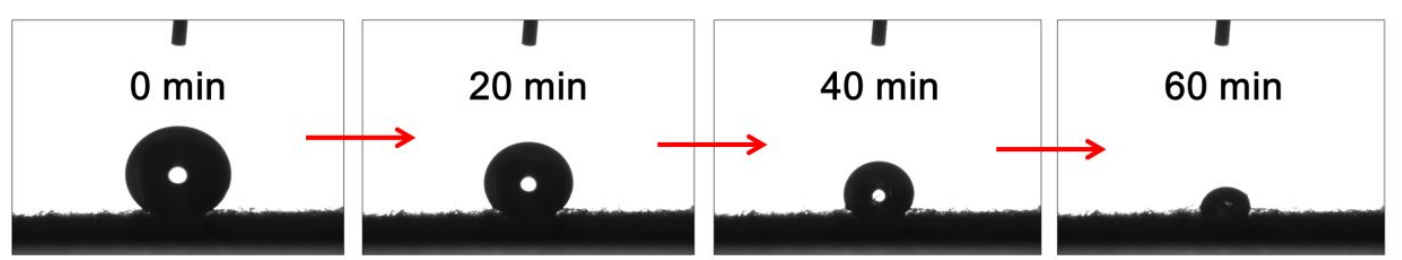

Figure S17. Water droplet $(5 \mu \mathrm{L})$ on $\mathbf{1}|\mathbf{A g N P s}|$ Cotton across a time span of 60 minutes under ambient conditions 


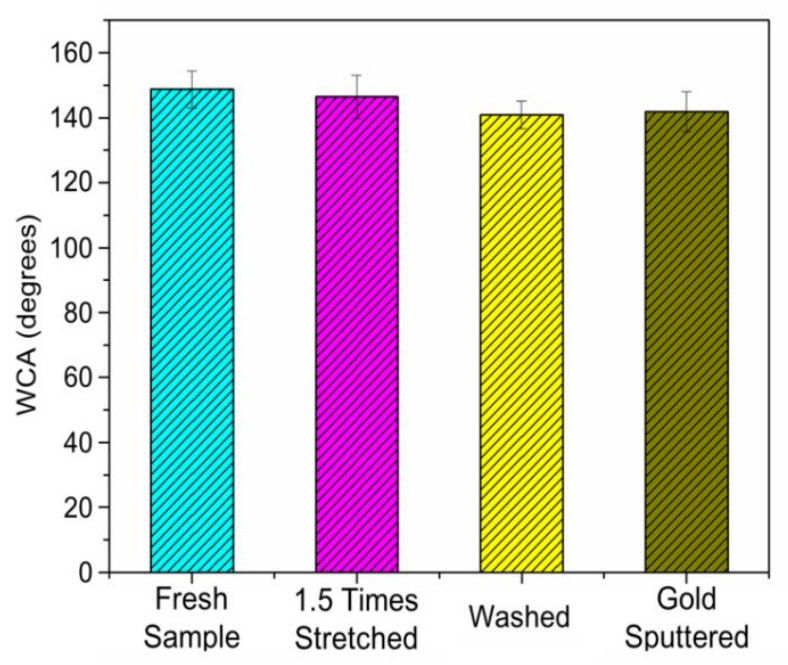

Figure S18. Comparison of WCA of $\mathbf{1}|\mathbf{A g N P s}|$ Cotton and that after various treatments

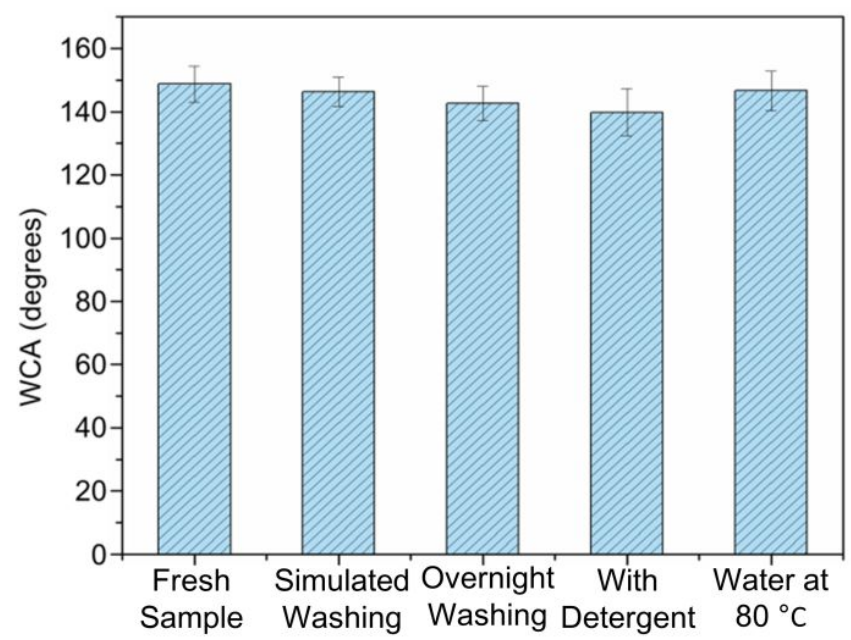

Figure S19. Comparison of WCA of $1|\mathbf{A g N P s}|$ Cotton and that after different washing conditions

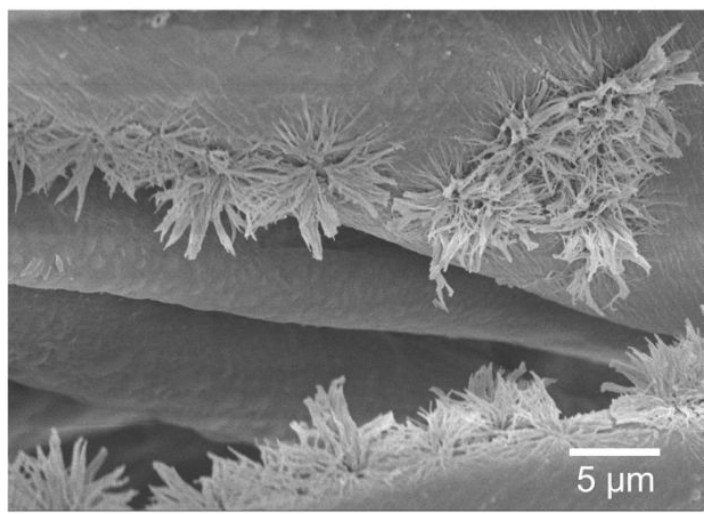

Figure S20. FESEM image of $\mathbf{1}|\mathbf{A g N P s}| \mathbf{C o t t o n}$ after the simulated washing treatment 


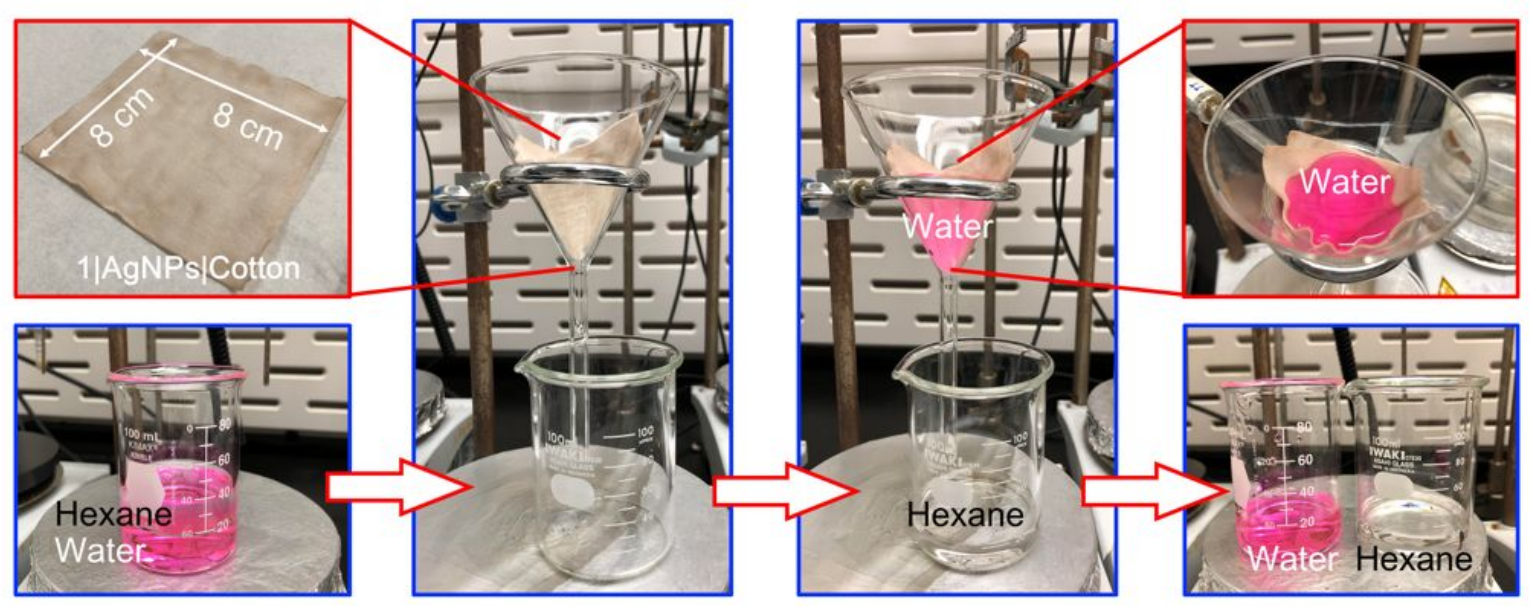

Figure S21. Demonstration of rapid water $/ n$-hexane separation by a simple filtrator constructed using $\mathbf{1}|\mathbf{A g N P s}| \mathbf{C o t t o n}$ 